\title{
A multi-disciplinary approach to weight management of school-age girls: a study protocol
}

Multidyscyplinarne podejście do kontroli masy ciała dziewcząt w wieku szkolnym protokół badania

\author{
${ }^{1}$ Sara Salahshornezhad, ${ }^{2}$ Zahra Sohrabi, ${ }^{3}$ Saeid Doaei, ${ }^{4}$ Maryam Gholamalizadeh, ${ }^{5}$ Arash Mani, \\ ${ }^{6}$ Manoosh Mehrabi, ${ }^{2}$ Morteza Zare, ${ }^{1,2}$ Marzieh Akbarzadeh
}

${ }^{1}$ Department of Community Nutrition, School of Nutrition and Food Sciences, Shiraz University of Medical Sciences, Shiraz, Iran

${ }^{2}$ Nutrition Research Center, School of Nutrition and Food Sciences, Shiraz University of Medical Sciences, Shiraz, Iran

${ }^{3}$ Research Center of Health and Enviroment, School of Health, Guilan university of Medical Sciences, Rasht, Iran

${ }^{4}$ Student Research Committee, Cancer Research Center, Shahid Beheshti University of Medical Sciences, Tehran, Iran

${ }^{5}$ Research Center for Psychiatry and Behavioral Sciences, Shiraz University of Medical Sciences, Shiraz, Iran ${ }^{6}$ Department of E-learning in Medical Sciences, Virtual school, Shiraz University of Medical Sciences,

Shiraz, Iran

\begin{abstract}
Background: There is lack of data about the combination of multiple approaches to control being overweight and obesity in adolescents. This study aimed to evaluate the effect of a combination program including smart-phone nutrition education, physical activity, and cognitive behavioral therapy (CBT) programs in the management of obesity and overweight among elementary school girls in comparison with a traditional education method.

Material and methods: The primary outcome of this study is the assessment of changes in anthropometric data after 10 weeks. The secondary outcome is investigating the effect of treatment on biochemical data. Sixty-two elementary school girls aged 9-12 years with the body mass index (BMI) for age above 85th percentile will be chosen and randomly assigned to one of the two groups of control or intervention. During the 10 weeks of the intervention, smartphone games will be given to the children with some information at the beginning about the causes and complications of obesity, food sources of fiber and vitamins, and banned foods during the intervention period. Students in the intervention group will participate in an aerobics exercise for 45 minutes three times per week under the supervision of an experienced trainer and eight CBT Sessions which were designed for children by a clinical psychologist. Anthropometric measurements, including height, weight, waist circumference (WC), hip circumference and waist to hip ratio(WHR), and biochemical and metabolic lab data, including fasting blood sugar (FBS), total cholesterol, high density lipoproteins (HDL), low density lipoproteins (LDL), leptin and will be measured at the baseline and 10 weeks later at the end of intervention period . Also, both questionnaires of metabolic equivalent test (MET) and Dutch Eating Behavior Questionnaire (DEBQ) will be completed for each student at baseline and after 10 weeks. The students in the control group will receive traditional education in the form of lectures given by a nutritionist regarding weight management.

Discussion: All of the results of this study will be published to supply information about the management of being overweight and obesity in children via multi-disciplinary approaches, including nutrition education with mobile-based health interventions, cognitive behavioral therapy, and physical activity.
\end{abstract}

\section{Key words:}

obesity, adolescent, cognitive behavioral therapy, mobile health, telemedicine. 


\section{Introduction}

Obesity is one of the most prevalent risk factors for chronic diseases around the world [1]. It is an important health concern with a dramatic rising prevalence in less developed countries [2]. According to the latest statistics of world health organization, 18\% of children and adolescents aged 5-19 were overweight or obese in 2016 [6]. Even during young ages, obesity can increase the risk of hypertension and dyslipidemia which may lead to speeding up the progression of atherosclerosis in adulthood [3]. The most important causes of obesity include genetic factors, socioeconomic factors, inactivity, inappropriate dietary habits, and psychological stress. Moreover, there is an association between weight gain and psychiatric morbidities, including depression, dissatisfaction with body image, and low self-esteem [4]. Hence, managing psychological stress could possibly be important in controlling being overweight and obesity in children. One of the methods for psychological management of obesity is a method called cognitive behavioral therapy (CBT) [5]. It is a kind of therapy that uses different techniques of behavioral therapy to control behaviors by changing antecedents and improving the consequences. This method mainly combines with cognitive techniques to make any changes in negative automatic thoughts (NAT), dysfunctional cognitions, and beliefs. CBT also uses strategies to promote the generalization and transfer of new skills and behaviors learned in therapy into everyday life and maintain during life span [6].

The comprehensive intervention programs focusing on different aspects of obesity are recommended for better management of obesity and being overweight [7]. This paper is aimed to evaluate the effect of a comprehensive protocol, including smart-phone nutrition education, physical activity, and CBT programs for the management of obesity and being overweight in elementary school girls in comparison with traditional education.

\section{Summary of protocol}

\section{Aim}

The primary aim of this randomized clinical trial is to evaluate the effect of a comprehensive weight reduction intervention including smartphone game applications and cognitive behavioral therapy plus physical activity compared to traditional education in the form of lectures given by a nutritionist regarding weight management on anthropometric indices, lipid and sugar profile, leptin level, and body composition of adolescent girls.

\section{Methods/design}

Trial design: It is a randomized controlled trial of 10 weeks.

Participants: 62 girls aged 9 to 12 years from randomly chosen schools of four districts of Shiraz, Iran, will be chosen according to the inclusion/exclusion criteria (Fig. 1).

\section{Eligibility criteria}

Inclusion criteria: School girls' age should be between 9 and 12 years. The reason for choosing this age group is that students should have enough literacy to do CBT homework. They should also be at the ages before puberty. The participants' percentile of body mass index for age should be above the $85^{\text {th }}$, and only students who are willing to take part in the study will be chosen after filling written informed consent by themselves and their parents. They will be reassured that they can leave the intervention whenever they want. These children will need to own a smart phone or tablet and be keen on smart phone games as well.

Exclusion criteria: All participants should fill children depression inventory (CDI) questionnaire before any intervention and experiment, and, accordingly, depressed children will be excluded. Any disease which needs a resting time or treatment during the study (taking medicines which affect weight specifically) will lead to exclusion, and children who already use any intervention for their being overweight or obesity would be excluded as well. Also, obesity with genetic, metabolic and hormonal causes is not targeted in this study.

\section{Sample size calculation}

Sample size was determined according to weight, as a primary outcome using the standard formula suggested for parallel-design randomized controlled trials [8]. A targeted sample size of 62 girls was determined with a mean difference of 0.2 kilograms and standard deviation of 1.5 for weight [8], considering the level of 0.05 , power of $85 \%$, and $20 \%$ dropout rate.

Sixty-two participants will be selected by simple random sampling from those eligible for participation and will be randomized into two groups. Thirty-one participants will be assigned to the intervention group, and 31 participants will be assigned to the control group [8].

\section{Ethical approval}

The study protocol is reviewed and approved by the Ethics Committee of Shiraz University of Medical Sciences (SUMS) and is conformed to the Declaration of Helsinki and Good Clinical Practice Guidelines. The study protocol is also available at Iranian Registry of Clinical Trials (www.irct.ir) under the identification number: IRCT20190508043531N1.

\section{Randomization}

An independent statistician who is not involved in data collection and analysis will do the allocation of eligible children using Balanced Blocked Randomization (BBR) with a fixed block size of four. It should be noted that all interdisciplinary researchers and children will be blinded to the group allocations, and the code assignments will not be announced until the statistical analyses are done.

\section{Outcomes}

The effectiveness of multi-disciplinary intervention in 9-12-year-old female students will be assessed by comparing intervention and control groups in:

a) Anthropometric indices: weight, height, body mass index, waist circumference, hip circumference, waist-to-hip ratio, and waist-to-height ratio at baseline and after 10 weeks as secondary outcomes. 


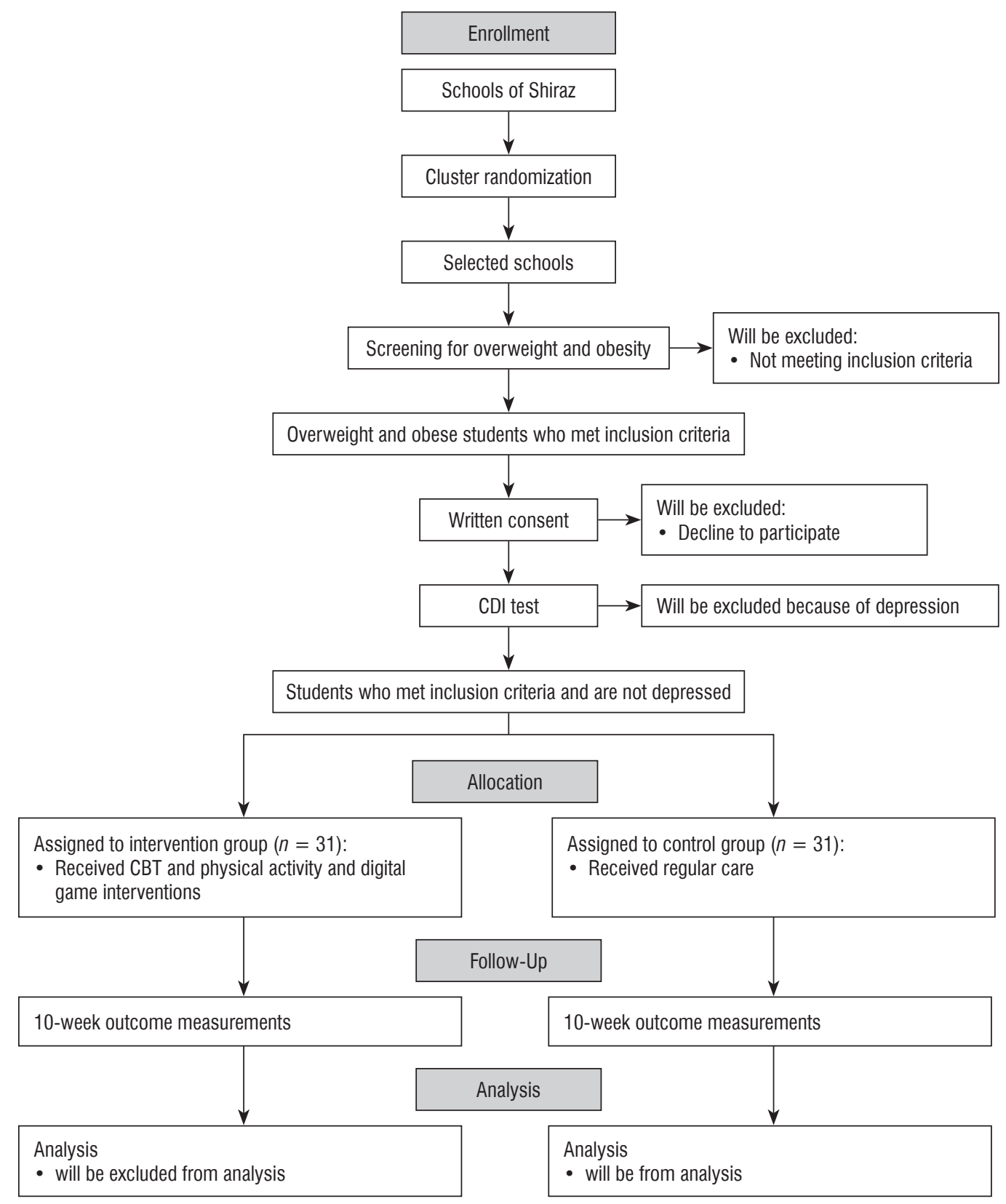

Figure 1. Flow diagram of the study

b) Body composition measurements, including the percentage of body fat and muscle mass at baseline and after 10 weeks as secondary outcomes.

c) Evaluations of dietary habits and physical activity as secondary outcomes: The results of DEBQ and MET questionnaires at the baseline and after 10 weeks.

e) The changes in serum lipids, fasting glucose, and serum leptin at the baseline and after 10 week as secondary outcomes.

\section{Outcome measurements}

Before and after the intervention, anthropometric measurements comprising height, weight, and waist and hip circumference will be done for all the students.

Individuals will be weighed on a Seca scale weighing at least $0.1 \mathrm{~kg}$ to a maximum of 150 kilograms with 10 grams error, with minimal coverage and without shoes in the morning before having breakfast. The scale will be calibrated before each day of measurement. Height will be measured two times to the near- 
est $0.1 \mathrm{~cm}$ with no shoes, using a Seca 222 stadiometer. Using the mean of these measurements, body mass index (BMI) will be calculated as weight in kilograms divided by height in square meters $\left(\mathrm{kg} / \mathrm{m}^{2}\right)$. Body mass index between the $85^{\text {th }}$ and $95^{\text {th }}$ percentiles is described as being overweight and equal to or greater than the $95^{\text {th }}$ percentile, as an obesity criterion. In this study, anthropometric measurements of students are compared with standard charts of the World Health Organization [9]

Following the protocol of the World Health Organization, waist circumference will be measured using a non-elastic but flexible measuring tape on the approximate midpoint in between the lower margin of the last palpable rib and the top of iliac crest [10]. Hip circumference will be measured in the most prominent part with a non-elastic measuring tape without imposing pressure on the body with $0.1 \mathrm{~cm}$ accuracy. WHR is calculated by dividing waist circumference by hip circumference. In order to eliminate individual errors, all measurements are performed by one person [10].

All anthropometric outcomes will be assessed at baseline and after 10 weeks.

\section{Muscle mass and body fat measurements}

Total body fat, lean body mass, total fat percentage, and lean body mass will be measured by Bioelectrical Impedance Analysis method (TANITA, IRAN). All body composition variables will be assessed at baseline and after 10 weeks (at the beginning and at the end of the study phase).

This device will give results on extracellular and intracellular water, weight, skeletal muscle mass, body fat mass, body mass index, body fat percentage, basal metabolism rate, visceral fat, and the amount of internal and external water in each organ.

For assessing body composition by the BIA, students will be asked to stand on the metal plates with bare feet, 4 hours after a light breakfast in the morning (no eating or drinking is allowed after that) and 12 hours after any types of vigorous exercise. Before the test, students will be asked about their levels of physical activity (which is a factor in estimating body composition for this machine as well as the height of students). Using this information which will be entered into the body analyzer, body composition and the percentages of body fat and body muscle will be estimated.

\section{Biochemical evaluations}

For biochemical evaluations and evaluations of serum variables, the subjects will be asked to go to Imam Reza Lab in Imam Reza clinic, Shiraz, Iran. Four ml of venous blood sample will be taken in a sitting position following a standard protocol for 8 hours of fasting. The serum will be centrifuged and transferred to the laboratory of the School of Nutrition and Food Sciences of Shiraz University of Medical Sciences until final measurements, and it is kept in the $-70^{\circ} \mathrm{C}$. Most biochemical variables, including blood lipids and sugar will be measured by an automated analyzer (Hitachi902, Roche, Japan) using kits by Pars Test by colorimetric method.

Total cholesterol (TC) and triglyceride (TG) levels will be measured by enzymatic calorimetry using cholesterol oxidase, esterase, and glycerol phosphate oxidase. Serum HDL-C levels are measured by the same method after deposition of apo-B containing lipoproteins.

The leptin hormone will be measured by Enzyme-linked Immune Sorbent Assay (ELISA) kits (IBL, Germany), which will be tested according to the standard procedure and control curves at a specific time.

\section{Questionnaires}

a) Demographic Evaluations:

Students' demographic characteristics will be assessed by a researcher-designed questionnaire. This questionnaire contains regular demographic information, including age, parents' job, parents' education, number of children, and the like.

b) Children Depression Inventory (CDI):

CDI questionnaire will be completed by the participants. If the child is depressed, she will be excluded from the study, and another child with appropriate entry criteria will be replaced. The CDI was developed by Cox and Beck to measure depression in children. The questionnaire consists of five subscales of negative mood, interpersonal problems, inadequacy, inattention, and negative self-esteem and is designed to focus on school assignments. The children will choose one of three sentences that expressed his or her feelings, thoughts, and behavior over the past two weeks. Questions are scored from 1 to 2 [11].

Dehshiri et al. investigated the reliability and validity of the Persian version of CDI (sampling group of 407 school children), and the results showed that the test-retest reliability was 0.82 , and the correlation coefficients between CDI and Children Depression Scale showed that CDI was valid [12].

c) MET Questionnaire:

This questionnaire collects proper items about type, frequency, and period of activities to evaluate the average physical activity level. Particularly, a MET is defined as the ratio of the metabolic rate divided by the resting metabolic rate (almost $1.0 \mathrm{kcal} / \mathrm{kg} /$ hour for adults) [13].

d) Dutch Eating Behavior Questionnaire

One aim of this study is to define the eating behaviors using Dutch's Eating Behavior Questionnaire. Halvarson et al. assessed the reliability of this questionnaire in the population of school girls (9-10-year-old) (reliability =0.83) [14]. A 2006 research in Shiraz, Iran, used the back translation method and validated this questionnaire [15].

The questionnaire consists of two parts. The first part of the questionnaire includes demographic information and characteristics related to the students' weight and height. The second part of the questionnaire consists of questions with three parts related to eating stimuli, external stimuli, and eating restriction [15].

\section{Intervention}

This study contains a school-based multi-component single-phase obesity treatment intervention for 9-12-year-old girls which concentrates on three main points:

1. Improving the quality of food choices which means reducing foods with high caloric density and increasing the consumption of fiber through eating more fruits, vegetables, whole 
grains, and legumes and also an increase in low fat and non-fat dairies and low-fat MFP (meat/fish/poultry) proteins by nutrition education trough using a smartphone game. The smartphone game is developed in collaboration with the Virtual School of Shiraz University of Medical Sciences. At the beginning of the game, some information about the causes and complications of obesity, number of calories in various foods, food sources of fiber, vitamins, minerals, and proteins, and banned foods during the intervention are presented to children in a video. In each game, the children can play for no more than 45 minutes. In this step, we mainly focus on nutrition education through a smartphone game.

2. Being aware during eating time through CBT Sessions:

The psychological intervention is organized as a group cognitive behavioral therapy in eight sessions in groups of ten. A clinical psychologist performs all the sessions in the school.

The protocol of these sessions contains the following steps:

Start with a warm-up that will break the kids' communication ice at a group meeting.

First session: (A) Emphasizing group therapy principles, such as confidentiality and striving for group integrity. (B) The collaborative work of the members working together on the basis of the ABC (Activating effect, Belief, Consequences) behavior training model. C) Preparing the participants to fill the $A B C$ table to eat over the next week

Second Session: Start treatment by examining the question 'Why do we overeat?'. The therapist checks the participants' patterns of overeating (Activating effect) and then gives a presentation on these issues and begins giving clues to clients. At the end of this session, participants will learn to recognize these acting effects and will be asked to control them as much as possible over the next week.

Third Session: A) Assignment Assessment. B) Examining the Causes of Failure.

The therapist will extract mood-based failure patterns and negative emotions, such as aggression and provide a lecture about emotion-coping strategies. Participants will know that we are eating instead of dealing with issues that are bothering us. The therapist will check for activities which children can do in such situations instead of eating for each participant individually. A list of solutions will be given to participants so that they can do something from the list or similar activities based on their creativity.

Fourth Session: A) Assignment Assessments. B) Review of Failures.

The therapist will relate the failure pattern to situations which lead the children to overeat in order to teach them to leave that situation. Participants will learn to eat in a fixed location, such as the kitchen so that any link between the place and/or a particular situation with overeating will break.

Fifth Meeting: Review what has been done so far and diagram children's progress in managing their eating behaviors with their own help and feedback.

Sixth Session: A) Checking the Causes of Failure. B) Reviewing the Disturbing Thoughts that Clients Have about their Success or Failure. C) The therapist will emphasize the chil- dren's thoughts and will teach them skills of behavior and thought controlling.

Seventh Session: The therapist continues to address the thoughts.

Eighth Session: There will be a summary and presentation of the cognitive behavioral model and training in skills necessary for relapse prevention.

3. Physical Activity Sessions:

During these ten weeks of intervention, three sessions of aerobics exercise each week for 45 minutes will be conducted by a master of sports physiology, followed by anthropometric measurements and laboratory data retrieval after ten weeks of intervention.

\section{Timing of assessment}

Children in the control group receive usual care and usual nutrition education about correcting dietary habits with traditional methods including lectures by a dietitian (the main investigator).

Children in both groups will be assessed twice; at the baseline and 10 weeks later at the end of the study phase.

\section{Discussion}

This paper portrays the protocol for a cluster randomized clinical trial to figure out the effectiveness of a multidisciplinary intervention. We designed this study to aim at treating obesity in children. The results from this study will provide evidence as to the effectiveness of a multi-disciplinary intervention which seeks to help to control children's obesity in schools engaging students, their parents, and school authorities.

In spite of the role of genetics in vulnerability to obesity, there is expanding insight into obesogenic habits which may lead to this epidemic of obesity-related metabolic diseases $[2,16]$.

The hypothesis of current study is that changes in the mean of anthropometric and biochemical metabolic factors differ between the intervention and control groups after a 10-week intervention period.

The ten-week intervention of smart phone nutrition and cognitive behavioral therapy plus regular physical activity 3 times per week could possibly normalize weight, body mass index, waist and hip circumference, waist to hip ratio, total cholesterol, LDL, HDL, serum triglyceride, and fasting blood sugar levels because the education in the smart phone will encourage them to avoid dietary sources saturated and trans fatty acids, such as fast foods, fries, desserts, etc. and change the type of their consumed carbohydrates from simple to complex. They will also learn to increase sources of dietary fibers which may lead to control blood sugar and total cholesterol. All the same, cognitive behavioral therapy will make them prepared to overcome their stress and consequently overeating (which in most cases happens as a response to stress) [8]. At the same time, the role of physical activity in controlling these indicators must not be forgotten. A loss in weight would cause differences in muscle mass and fat mass which leads to changes in serum leptin. 
The main potencies of the study are as follows: extensive information about the effect of improvement in health markers and health-concerned habits can be obtained via questionnaires, anthropometric data, bioelectrical impedance analysis, and biochemical and metabolic factors, plus assessing the effect of a multi-disciplinary intervention in childhood obesity.

The authors found no studies that considered the simultaneous association of psychological intervention and physical activity alongside nutrition education smart phone game in this particular age group, and no information was available in this field. The results of the current study will give us more insight into the effects of simultaneous utilization of these interventions on anthropometric and biochemical metabolic factors of elementary school girls.

Another strength of this study is the use of a smart phone game to teach nutrition. Children are able to learn in various ways, and, nowadays, the importance of online education is undeniable. This method might be even more encouraging in the form of a smart phone game. Health-concerned habits and behaviors may be improved through using smart phone games [17].

\section{References}

1. Kumar S, Kelly AS. Review of Childhood Obesity: From Epidemiology, Etiology, and Comorbidities to Clinical Assessment and Treatment. Mayo Clin Proc 2017; 92: 251-265. doi: 10.1016/j. mayocp.2016.09.017.

2. Hajian-Tilaki K, Heidari B. Prevalences of overweight and obesity and their association with physical activity pattern among Iranian adolescents aged 12-17 years. Public Health Nutr 2012; 15: 2246 2252. doi: $10.1017 / S 1368980012001048$.

3. Basiratnia M, Derakhshan D, Ajdari S, Saki F. Prevalence of childhood obesity and hypertension in south of Iran. Iran J Kidney Dis 2013; 7: 282-289.

4. Grube M, Bergmann S, Keitel A, et al. Obese parents - obese children? Psychological-psychiatric risk factors of parental behavior and experience for the development of obesity in children aged 0-3: study protocol. BMC Public Health 2013; 13: 1193. doi: 10.1186/1471-2458-13-1193.

5. Wilfley DE, Kolko RP, Kass AE. Cognitive-behavioral therapy for weight management and eating disorders in children and adolescents. Child Adolesc Psychiatr Clin N Am. 2011; 20: 271-285. doi: 10.1016/j.chc.2011.01.002.

6. https://www.who.int/gho/ncd/risk_factors/overweight_obesity/overweight_adolescents/en/

7. Rey-López JP, Vicente-Rodríguez G, Biosca M, et al. Sedentary behaviour and obesity development in children and adolescents. Nutr Metab Cardiovasc Dis 2008; 18: 242-251.

8. Tsiros MD, Sinn N, Brennan L, et al. Cognitive behavioral therapy improves diet and body composition in overweight and obese adolescents. Am J Clin Nutr 2008; 87: 1134-1140.
On the other hand, all anthropometric measurements will be performed by a nutritionist with the same and calibrated instruments and these measurements of students will be performed individually in a private environment. Necessary training to comply with the conditions of biochemical tests will be provided to the parents of students.

Parents of the students in the control group will also be suggested to receive the game if the outcomes are desirable for weight management.

In summary, this study looks for gaining perception and awareness into the lifestyle, habits and health status of children and ways for managing obesity and being overweight in this age group.

\section{Acknowledgments}

The authors gratefully acknowledge the children and their parents who participated in this study. This article is extracted from a master thesis done by Sara Salahshoornezhad with the grant number of "01-84-16675".

9. https://www.who.int/growthref/who2007 bmi for age/en/

10. https://apps.who.int/iris/bitstream/handle/10665/44583/978924150 1491_eng.pdf?sequence $=1$.

11. Rofey DL, et al. Cognitive-behavioral therapy for physical and emotional disturbances in adolescents with polycystic ovary syndrome: a pilot study. Journal of Pediatric Psychology 2009; 34: 156-163.

12. Dehshiri GR, Najafi M, Shikhi M, et al. Investigating Primary Psychometric Properties of Children's Depression Inventory (CDI). Journal of Family Research 2009; 5: 159-177.

13. Littman AJ, White E, Kristal AR, et al. Assessment of a one-page questionnaire on long-term recreational physical activity. Epidemiology 2004; 15: 105-113.

14. The difference in eating behavior among Italian normal weight, overweight and obese adolescent. Behavior Therapy and Research 2004; 58: 1217-1222.

15. Sarvestani RS, Kargar M, Kaveh M, Tabatabaei H. Effect of behavior modification programme on eating behaviors in obese adolescent female student. Iranian Journal of Nursing Research 2007; 2 : 13-18

16. Hajian Tilaki K, Sojjodi P, Razavi A. Prevalence of overweight and obesity and associated risk factors in urban primary-school children in Babol, Islamic Republic of Iran. EMHJ-Eastern Mediterranean Health Journal 2011; 17: 109-114.

17. O'Malley $G$, Clarke M, Burls A, et al. A smartphone intervention for adolescent obesity: study protocol for a randomised controlled non-inferiority trial. Trials 2014; 15: 15-43. 